\title{
Neuroprotective effect of insulin-loaded chitosan nanoparticles/PLGA-PEG-PLGA hydrogel on diabetic retinopathy in rats
}

This article was published in the following Dove Medical Press journal: International Journal of Nanomedicine

\author{
Xianfang Rongl-5 \\ Yinghong $\mathrm{Ji}^{1-5}$ \\ Xiangjia Zhul-5 \\ Jin Yang ${ }^{1-5}$ \\ Dongjin Qian ${ }^{1-5}$ \\ Xiaofen $\mathrm{Mo}^{1-5}$ \\ Yi Lu ${ }^{1-5}$
}

'Department of Ophthalmology and The Eye Institute, Eye and Ear, Nose, and Throat Hospital, Fudan University, Shanghai, China; ${ }^{2}$ The Key Laboratory of Myopia, Ministry of Health, Shanghai, China; ${ }^{3}$ Shanghai Key Laboratory of Visual Impairment and Restoration, Shanghai, China; ${ }^{4}$ Key National Health Committee Key Laboratory of Myopia, Fudan University, Shanghai, China; ${ }^{5}$ Laboratory of Myopia, Chinese Academy of Medical Sciences, Shanghai, China
Correspondence: Xiaofen Mo; Yi Lu Eye Institute, Eye and Ear, Nose, and Throat Hospital of Fudan University, 83 Fenyang Road, Shanghai 20003I, China Tel/fax +86 2l 6437 7l34 Email xfmo@fudan.edu.cn; luyieent@।26.com
Background: To pursuit effective sustained release systems for insulin to treat diabetic retinopathy (DR), a novel insulin delivering system was developed via loading onto chitosan nanoparticles/poly(lactic-co-glycolic acid)-poly(ethylene glycol)-poly(lactic-co-glycolic acid) hydrogel (ICNPH).

Methods and materials: Examinations including electroretinography, HE staining, transmission electron microscopy, terminal deoxynucleotidyl transferased UTP nick-end labeling, immunofluorescence, Western blot, and real-time polymerase chain reaction were performed to evaluate the neuroprotective efficacy of ICNPH on DR by a single subconjunctival injection. Results: Compared with the insulin, blank, and sham treatment groups, subconjunctival injection of ICNPH significantly reduced the decrease of scotopic B-wave amplitude, alleviated retinal micro- and ultrastructural changes, and reduced retinal cell apoptosis caused in DR rats. Meanwhile, a significant reduction of vascular endothelial growth factor and glial fibrillary acidic protein expression as well as a remarkable increase in Occludin expression was also found in retinas in ICNPH group compared with the sham treatment group.

Conclusion: The results indicate that ICNPH has sufficient neuroprotective effect on retinas through subconjunctival injection in DR rats and facilitates controlled insulin delivery. It might be one of the therapeutic strategies for DR in the near future.

Keywords: ICNPH, diabetic retinopathy, subconjunctival injection, insulin delivery, thermosensitive hydrogel, PLGA-PEG-PLGA hydrogel

\section{Introduction}

Diabetic retinopathy (DR), a common serious microvascular complication of diabetes, is one of the leading causes of visual impairment and blindness among adults, leading to detrimental effects on the quality of life of more than half of the diabetes patients worldwide. ${ }^{1}$ Although maintenance of the basal insulin level in diabetic subjects has been achieved to an extent, the medicinal properties of the drugs employed are greatly weakened as a result owing to limitations of the traditional drug delivery methods. For instance, although systemic insulin therapy reduces the risk of microvascular complications, ${ }^{2}$ it is also associated with increased probability of $\mathrm{DR}^{3}$ as well as high occurrence rates of hypoglycemia, hypoglycemic coma, weight gain, and ketoacidosis. ${ }^{4}$ Acute hypoglycemia induced by insulin treatment generally contributes to subsequent decline of visual function. ${ }^{5}$ Moreover, diabetic patients require insulin injections at least once a day owing to its rapid degradation rate and extremely short half-life, causing considerable pain and reduced quality of life.

Sustained release technology for insulin provides a favorable approach for local long-term administration to eyes. To date, several insulin delivery approaches, 
including nanoparticles, ${ }^{6}$ microspheres, ${ }^{7}$ and biodegradable hydrogels, have been explored to treat retinal diseases. ${ }^{8}$ Among the available drug delivery systems, biodegradable hydrogels based on both natural and synthetic polymers appear to be the most attractive option owing to several beneficial properties, including easy administration, localized delivery, and sustained release. ${ }^{9}$ However, these materials have a number of shortcomings since multiple implantations via frequent surgery are required to obtain the desired therapeutic effect, causing considerable damage to the eyeball, which limits their application in the clinic. In recent years, research groups have focused on the development of thermosensitive polymers capable of responding to temperature $\left(25^{\circ} \mathrm{C}\right) .{ }^{10} \mathrm{Such}$ "intelligent" polymers can flow freely at room temperature (RT) and achieve sol-gel transition at body temperature $\left(37^{\circ} \mathrm{C}\right)$. Drug loading on this system is accomplished by mixing drug and copolymer solutions, which are easily syringe-injected into specific body sites. The hydrogel formed is able to release the incorporated drugs continuously into the injected site as it biodegrades. Based on the injectability and solution-to-gel transition properties, thermosensitive hydrogels composed of diversified polymers are thus widely used as a drug delivery system. ${ }^{11-13}$

In this study, we developed a novel two-phase insulin sustained release system involving insulin-loaded chitosan nanoparticles entrapped in a poly(lactic-co-glycolic acid)poly(ethylene glycol)-poly(lactic-co-glycolic acid) (PLGAPEG-PLGA) hydrogel, which combines the advantages and avoids the flaws of the hydrogel and nanoparticle delivery systems. ${ }^{14,15}$ The novel thermosensitive insulin nanoparticle hydrogel-sustained release system (insulin-loaded chitosan nanoparticles/PLGA-PEG-PLGA hydrogel [ICNPH]) was injected once into subconjunctiva of diabetic rats and retinal histological and functional changes were evaluated at the designated time points. The major objective of this investigation was to determine the neuroprotective effects of ICNPH on DR, and to provide an evidence for its potential clinical application for DR in the future.

\section{Materials and methods}

\section{Preparation of insulin-loaded chitosan nanoparticles}

An appropriate amount of chitosan powder (molecular weight [MW] 340 kDa, 95\% deacetylation degree; Xingcheng Biomaterial Co., Ltd, Nantong, China) was dissolved in $1 \%$ acetic acid solution. After stirring at 1,000 rpm for 4 hours, chitosan solution $(10 \mathrm{mg} / \mathrm{mL})$ was prepared. A total of $5 \mathrm{~mL}$ chitosan solution was introduced directly into 0.1\% insulin (Wan-bang Biochemical Pharmaceutical Co.,
Ltd., Xuzhou, China) solution at an insulin: chitosan ratio of 1:15 under agitation. We slowly dropped 1\% triphenyl phosphate into the above mixture under stirring until the opalescence appeared to generate the insulin chitosan nanoparticle suspension. After centrifugation at 1,500 rpm for 10 minutes, the suspension was washed using deionized water and freeze-dried. The dry powder obtained was incubated at $-20^{\circ} \mathrm{C}$ until further use. The insulin-loaded chitosan nanoparticles generated were designated ICN, and particle size and morphology were determined using a Philips CM120 transmission electron microscope (TEM; Philips Electronics Ltd., Amsterdam, the Netherlands). The insulin content in chitosan nanoparticles suspension was measured via HPLC. After detection, the average size of the prepared nanoparticles was determined as $\sim 200 \mathrm{~nm}$ and total bonding efficacy of insulin in ICN as $\sim 12 \%$.

\section{Preparation of insulin-loaded chitosan nanoparticles/PLGA-PEG-PLGA hydrogel} Typically, PLGA-PEG-PLGA triblock copolymer (15001000-1500; Daigang Biomaterial Co., Ltd., Jinan, China) thermosensitive hydrogel was dissolved in distilled water at RT to obtain homogeneous liquid PLGA-PEG-PLGA solution at a concentration of $25 \%$. Next, forearmed insulinloaded chitosan nanoparticles were incorporated into the copolymer solution at a nanoparticle: hydrogel ratio of 1:20 at RT under mild stirring until the solution became transparent. The solution incorporating insulin nanoparticles was incubated at $37^{\circ} \mathrm{C}$ and a hydrogel formed. The prepared insulin-loaded chitosan nanoparticles/PLGA-PEG-PLGA hydrogel, designated ICNPH, exhibited a liquid state and good liquidity at $\leq 25^{\circ} \mathrm{C}$ while converting from a sol to gel state when the temperature exceeded $25^{\circ} \mathrm{C}$. Moreover, insulin was released from ICNPH over a 60 -day period continuously rather than in significant bursts.

\section{Animal experiments}

All animal experiments complied with the guidelines of the Association for Research in Vision and Ophthalmology and were approved by the Animal Care and Use Committee of Fudan University (Shanghai, China). Healthy adult SD rats weighing $200 \mathrm{~g}$ were obtained from the Medical Center of Fudan University. After a 12-hour fast period, animals received an intraperitoneal injection of $65 \mathrm{mg} / \mathrm{kg}$ streptozotocin (Sigma Chemical Co., St Louis, MO, USA) in $0.5 \mathrm{M}$ sodium citrate buffer, $\mathrm{pH}$ 4.5. Control nondiabetic rats were injected with citrate buffer alone. After 24 hours, rat blood glucose level $>16.7 \mathrm{mmol} / \mathrm{L}$ was considered to indicate successful establishment of the diabetic rat model. 
For in vivo experiments, rats were randomly divided into five groups (six rats/group): 1) Normal (control group): rats were not injected with any drugs; 2) ICNPH: diabetic rats received a single subconjunctival injection of $20 \mu \mathrm{L} \mathrm{ICNPH}$ containing $80 \mu \mathrm{g}$ insulin; 3) Insulin: diabetic rats received a single subconjunctival injection of $80 \mu \mathrm{g}$ insulin in $20 \mu \mathrm{L}$ deionized water; 4) Blank: diabetic rats received a single subconjunctival injection of $20 \mu \mathrm{L}$ blank nanosized hydrogel; 5) False injection (sham): an empty syringe with no drugs was punctured into conjunctiva of diabetic rats. Blood glucose levels were monitored at the designated times using Accucheck-Instant (Boehringer Mannheim, Indianapolis, IN, USA). Animals were subjected to subconjunctival injection at 4 weeks after successful establishment of the diabetic model and sacrificed 2 weeks after this time point. Eyeballs were collected for subsequent experiments.

\section{Electroretinography}

Rats in different groups were initially dark-adapted overnight for the scotopic response. Animals used for the electroretinography (ERG) test received a subconjunctival injection in the right eye, with the untouched left eye serving as a control. Before recording of ERG, all animals were anesthetized via intramuscular injection. ERG was assessed in different groups at 4 weeks after subconjunctival injection. Briefly, two gold cup electrodes covered with $2 \%$ methylcellulose were placed on both corneas and a needle electrode inserted into the back. Visual stimuli were produced from $5 \mathrm{~ms}$ flashes of white light. The responses to light flashes $\left(0.01 \mathrm{~cd} \cdot \mathrm{s} / \mathrm{m}^{2}\right.$ in scotopic ERG) from a photic stimulator were amplified, filtered, and averaged. Scotopic A-wave and B-wave amplitudes were, respectively, measured based on the differences in amplitude between recording at onset to the trough of negative deflection and the trough of the A-wave to the peak of the B-wave. All examinations were performed at RT and animals displayed no obvious tachypnea.

\section{Hematoxylin and eosin staining}

Eyeball samples were fixed in 4\% paraformaldehyde for 1 hour and placed in neutral formalin overnight. After dehydration via an ethanol gradient, eyeballs were embedded in paraffin and cut into $5 \mu \mathrm{m}$-thick sections. Paraffin-embedded sections encapsulated with optic nerve were reserved for HE staining. Microstructural retinal changes were examined under a microscope (Leica DM4000B; Leica Microsystems).

\section{Transmission electron microscopy}

Retinal ultrastructure in animals from different groups was examined under a TEM. First, the eyecups were removed and fixed in 3\% glutaraldehyde for 10 minutes. The retina was stripped from the eyecups and immersed in 3\% glutaraldehyde for 24 hours, followed by fixing in osmium tetroxide for 1 hour. After dehydration, embedding, and slicing, $70 \mathrm{~nm}$-thick sections were stained with hydrogen peroxide or acetic acid lead nitrate. Ultrastructural changes were examined using TEM (Philips Electronics Ltd., Amsterdam, the Netherlands).

\section{Terminal deoxynucleotidyltransferase- mediated dUTP nick end labeling}

Terminal deoxynucleotidyl transferased UTP nick-end labeling (TUNEL) staining was applied to observe apoptosis of retinal cells. The eyecups containing retinal tissues were extracted. After dehydration in graded sucrose solution at $4{ }^{\circ} \mathrm{C}$ for 12 hours, the anterior segments of eyecups were removed, following which eyecups were embedded in optimal cutting temperature compound at $-20^{\circ} \mathrm{C}$. Eyecups were sliced into sections of $10 \mu \mathrm{m}$ thickness, fixed in $4 \%$ paraformaldehyde for 20 minutes at RT, washed with PBS for 30 minutes, and immersed in $0.1 \%$ sodium citrate buffer containing $0.1 \%$ Triton X-100 at $4{ }^{\circ} \mathrm{C}$ for 2 minutes. After drying, TUNEL staining was performed using an in situ apoptosis detection kit (Calbiochem ${ }^{\circledR}$; MerkKGaA, Darmstadt, Germany) according to the manufacturer's recommendations. Apoptosis of retinal nerve cells was observed under a fluorescent microscope (Leica DM4000B).

\section{Immunofluorescence}

Retina sections were blocked in $10 \%$ goat serum with $0.3 \%$ Triton for 1 hour, followed by incubation with primary antibodies diluted in blocking solution (1:500) overnight at $4^{\circ} \mathrm{C}$. The primary antibodies used included mouse anti-glial fibrillary acidic protein (GFAP; Roche Diagnostics, Indianapolis, IN, USA), mouse anti-vascular endothelial growth factor (VEGF; Roche Diagnostics), and rabbit anti-Occludin (Zymed, San Francisco, CA, USA). After washing in PBS three times, retina sections were incubated with secondary antibody for 24 hours at $4^{\circ} \mathrm{C}$ and coverslipped using Fluoroshield with DAPI (Sigma Chemical Corp.). Specimens were observed under a confocal laser scanning microscope (Leica DM4000B).

\section{Western blot}

Western blot was performed to assess the protein expression levels of GFAP, VEGF, and Occludin. Briefly, rat retinas were lysed in RIPA (25 mmol/L Tris, $\mathrm{pH} 7.4,1 \mathrm{mmol} / \mathrm{L}$ EDTA, 0.1\% SDS, 1\% NP-40, $150 \mathrm{mmol} / \mathrm{L} \mathrm{NaCl}$, $0.5 \%$ deoxycholic acid, $1 \mathrm{mmol} / \mathrm{L}$ phenylmethylsulfonyl 
fluoride, $1 \mathrm{~g} / \mathrm{mL}$ aprotinin, $1 \mathrm{~g} / \mathrm{mL}$ leupeptin) containing proteinase inhibitors (Complete Mini; Roche Diagnostics). Protein samples $(30 \mathrm{mg}$ ) were electrophoresed via SDSPAGE and transferred onto nitrocellulose membranes. Next, membranes were incubated at $4^{\circ} \mathrm{C}$ overnight with antibodies specific for GFAP, VEGF, and Occludin (1:200 dilution; Santa Cruz Biotechnology) followed by peroxidase-labeled antibody (1:5,000 dilution) for 1 hour at RT. After washing in TBST, protein bands were visualized via enhanced chemiluminescence (Santa Cruz Biotechnology). Membranes incubated with antibodies against actin (Sigma) were used to assess equal protein loading.

\section{Real-time PCR}

The mRNA levels of GFAP, VEGF, and Occludin were measured via real-time polymerase chain reaction (PCR). Total RNA was extracted from tissues using the QIAGEN RNeasy Mini kit according to the manufacturer's instructions. cDNA was synthesized from mRNA using reverse transcription reagent (TransGen Biotech, Inc., Beijing, China) and stored at $-20^{\circ} \mathrm{C}$ for subsequent experiments. Specific primers were designed using Primer Premier version 6.0 software and synthesized by Sangon Biotech (Shanghai) Co., Ltd. (Shanghai, China). The primers employed were as follow: GFAP (forward: 5'-GCTCCAAGATGAAACCAACC-3'; reverse: 5'-CCTCCAGCGACTCAACCTT-3'), VEGF (forward: 5'-CTGCTGTGGACTTGAGTTGG-3'; reverse: 5'-CAAAC AGACTTCGGCCTCTC-3'), Occludin (forward: 5'-CCTGT CTATGCTCGTCATCG-3'; reverse: 5'-TAGCCGTAACC GTAGCCGTA-3') and the internal control, $\beta$-actin (forward: 5'-GTCTTCCCCTCCATCGTG-3'; reverse: 5'-AGGGTCAGGATGCCTCTCTT-3'). PCR was performed under the following conditions: 40 cycles at $95^{\circ} \mathrm{C}$ for 30 seconds, $95^{\circ} \mathrm{C}$ for 5 seconds, and $60^{\circ} \mathrm{C}$ for 30 seconds. The mRNA levels of target genes in each experimental group were calculated from the $2^{-\Delta \Delta \mathrm{Ct}}$ value.

\section{Statistical analysis}

Statistical analyses were conducted using the software SPSS version 18.0 (SPSS Inc., Chicago, IL, USA) and the results were depicted as mean $\pm \mathrm{SD}$. The differences among groups were determined via one-way analysis of variance. Data were considered significant at $P$-values $<0.05$.

\section{Results}

\section{Electroretinography findings}

The ERG test was performed to examine retinal function changes in DR rats at 4 weeks post subconjunctival injection.
Data from comparative analyses of scotopic ERG amplitudes between injected and intact eyes are presented in Figure 1. In the normal group, good symmetry of ERG amplitudes (A- and B-waves) was observed in both eyes while the B-wave of ERG was significantly decreased in all other groups. In the ICNPH group, scotopic B-wave amplitude was significantly higher compared with the sham group. However, limited or no changes in B-waves were observed in all insulin-, blank-, and sham-injected eyes, compared with the sham group.

\section{HE staining and TEM analyses for retinal microstructure}

HE staining and TEM were applied to examine the retinal microstructure and potential ultrastructural changes at 2 weeks post subconjunctival injection. HE staining revealed alterations in retinal thickness and the cell layer, as depicted in Figure 2A. Thick retinal tissue, intact structure, and closely arranged cells were observed in rats from the normal group while both retinal thickness and cell layers decreased significantly in DR rats in sham group. In the ICNPH treatment group, slight changes in retinal microstructure were observed compared with the normal control, while in the insulin and blank groups, retinal thickness and cell layers were remarkably decreased, similar to the sham group.

Ultrastructural changes in retinal tissues were examined via TEM (Figure $2 \mathrm{~B}$ and $\mathrm{C}$ ). In normal retinas, mitochondria displayed an intact structure with no obvious cell abnormalities in the vascular wall, the basal membrane was intact, and retinal ganglion cells (RGCs) were unwound. In retinas from the ICNPH-injected group, no cytoplasmic swelling or structural rupture of retinal vessels and ganglion cells was detected although the appearance of mitochondria in RGCs was swollen, compared with that in normal retina. In the other subconjunctival-injected groups, obvious retinal ultrastructural abnormalities were observed, such as swelling of retinal microvascular and basement membrane cells. Additionally, swelling of soma, intracellular organelles, and cytoplasm were detected in RGCs.

\section{TUNEL staining for cell apoptosis}

To determine the effect of ICNPH on retinal cell apoptosis induced by hyperglycemia, TUNEL assay was performed. No TUNEL-positive cells were present in layers of normal retina while apoptotic cells were detected at 2 weeks post subconjunctival injection in retinas of all diabetic rats (Figure 3). The number of TUNEL-positive cells in the ICNPH group was markedly lower than that in other injected groups, while no significant differences were showed 

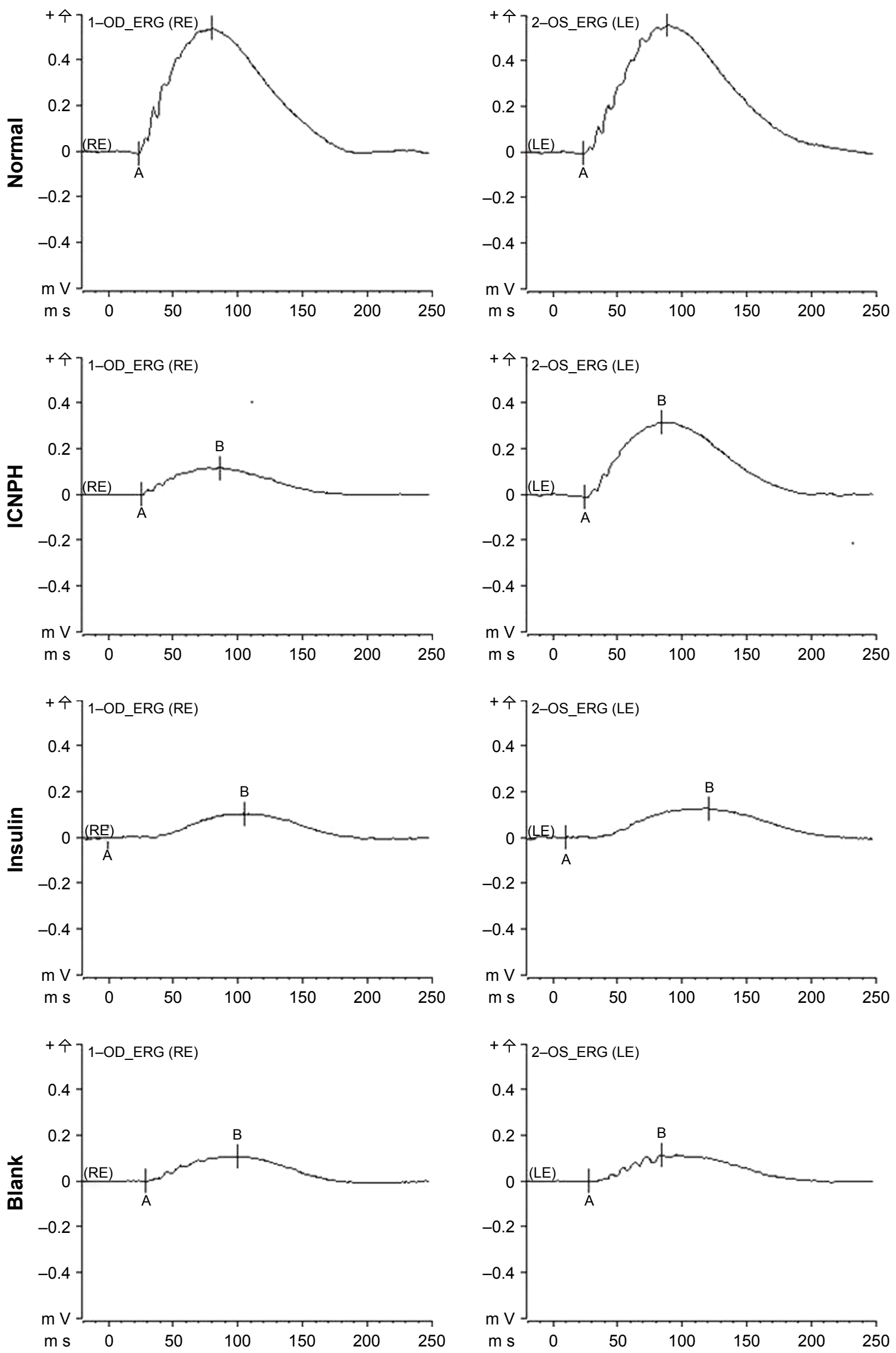

Figure I (Continued) 

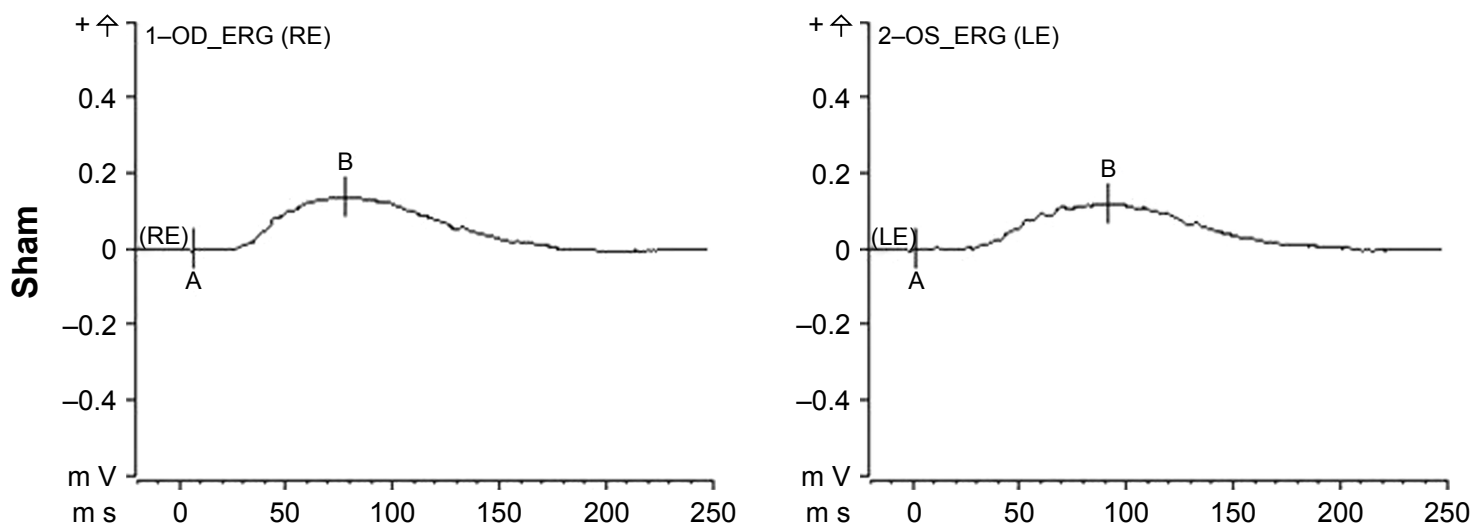

Figure I Scotopic ERG in rats at 4 weeks after subconjunctival injection.

Notes: Normal group: no injection with any drugs; ICNPH group: subconjunctival injection of $20 \mu \mathrm{L}$ ICNPH containing $80 \mu g$ insulin; Insulin group: subconjunctival injection of $80 \mu \mathrm{g}$ insulin in $20 \mu \mathrm{L}$ deionized water; Blank group: subconjunctival injection of $20 \mu \mathrm{L}$ blank nanosized hydrogel; Sham group: an empty syringe without any drugs was punctured into the conjunctiva of the diabetic rats.

Abbreviations: A, A-wave of the ERG; B, B-wave of the ERG; ERG, electroretinography; ICNPH, insulin-loaded chitosan nanoparticles/PLGA-PEG-PLGA composite hydrogel; LE, left eye; PLGA-PEG-PLGA, poly(lactic-co-glycolic acid)-poly(ethylene glycol)-poly(lactic-co-glycolic acid); RE, right eye.

A

Normal

ICNPH

Insulin

Blank

Sham
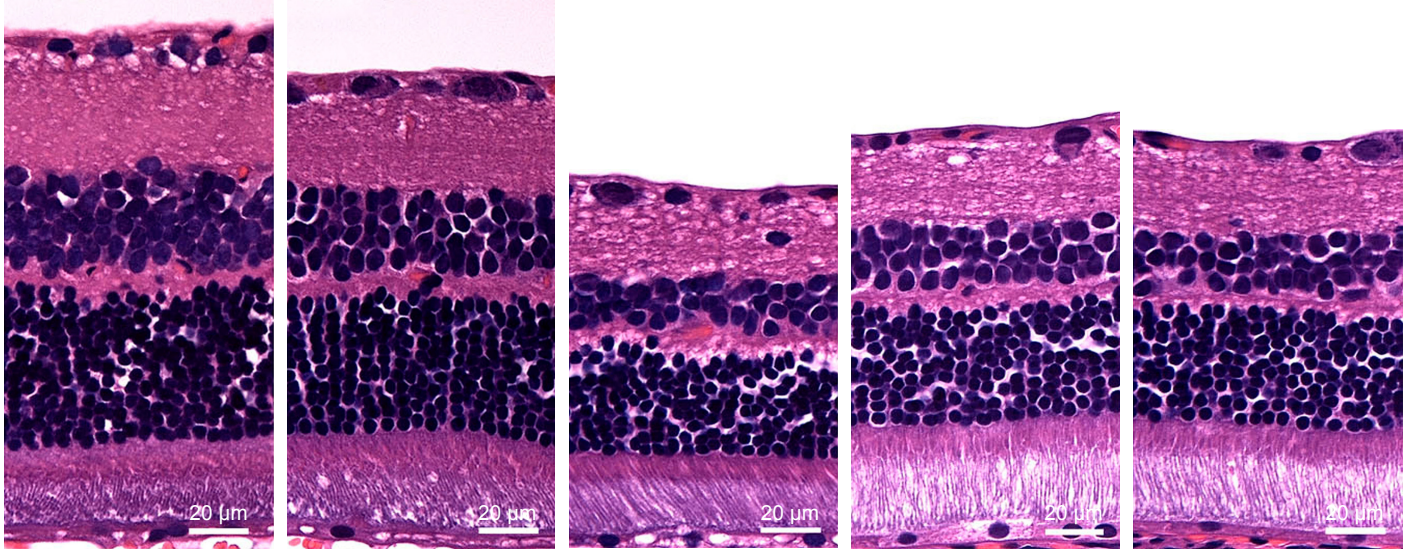

B
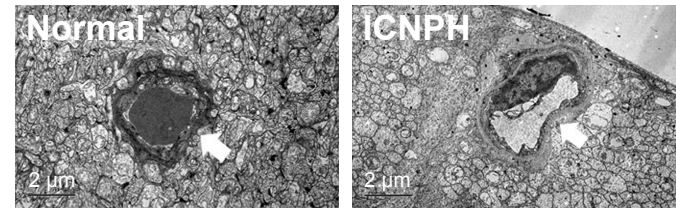

C
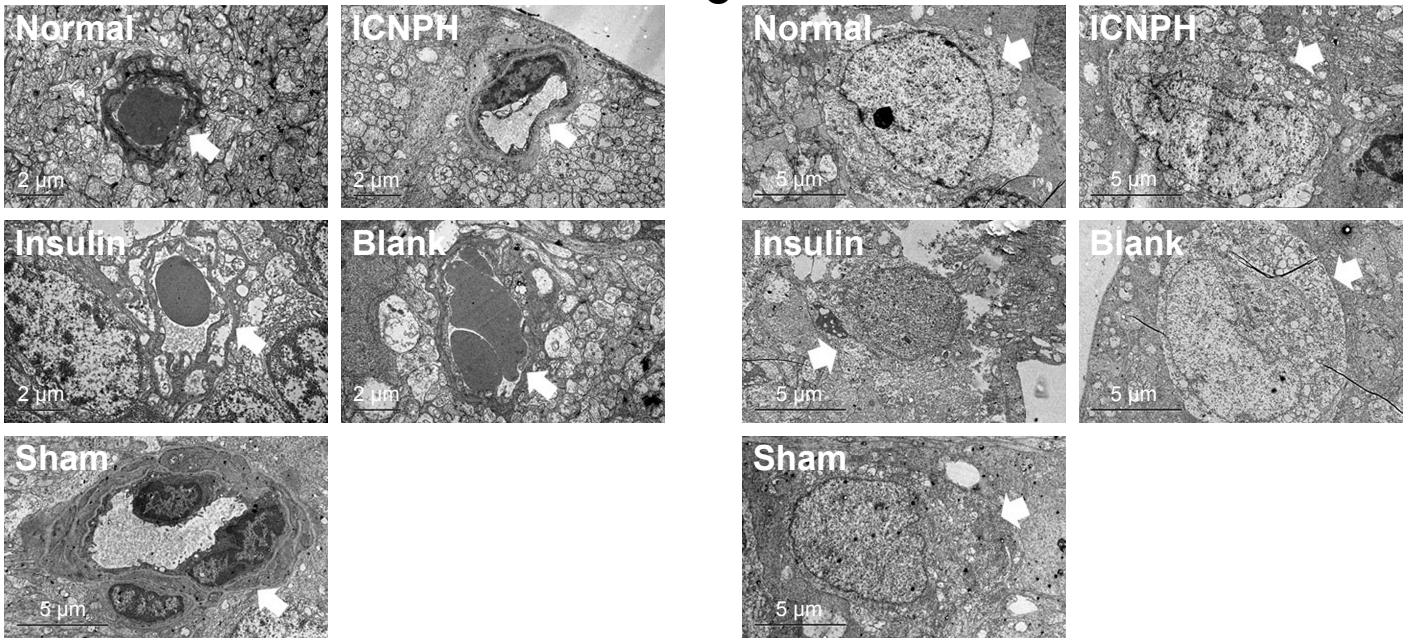

Figure $2 \mathrm{HE}$ staining and TEM observation of the retinal microstructure and ultrastructure changes in rats at 2 weeks after subconjunctival injection.

Notes: (A) The representative images of HE staining in retina in different groups. (B) The changes of retinal microvascular structure in different groups. (C) The changes of retinal ganglion cells structure in different groups. Retinal microvascular or retinal ganglion cells are identified by arrows. Normal group: no injection with any drugs; ICNPH group: subconjunctival injection of $20 \mu \mathrm{L} \mathrm{ICNPH}$ containing $80 \mu \mathrm{g}$ insulin; Insulin group: subconjunctival injection of $80 \mu \mathrm{g}$ insulin in $20 \mu \mathrm{L}$ deionized water; Blank group: subconjunctival injection of $20 \mu \mathrm{L}$ blank nanosized hydrogel; Sham group: an empty syringe without any drugs was punctured into the conjunctiva of the diabetic rats.

Abbreviations: ICNPH, insulin-loaded chitosan nanoparticles/PLGA-PEG-PLGA composite hydrogel; PLGA-PEG-PLGA, poly(lactic-co-glycolic acid)-poly(ethylene glycol)poly(lactic-co-glycolic acid); TEM, transmission electron microscope. 

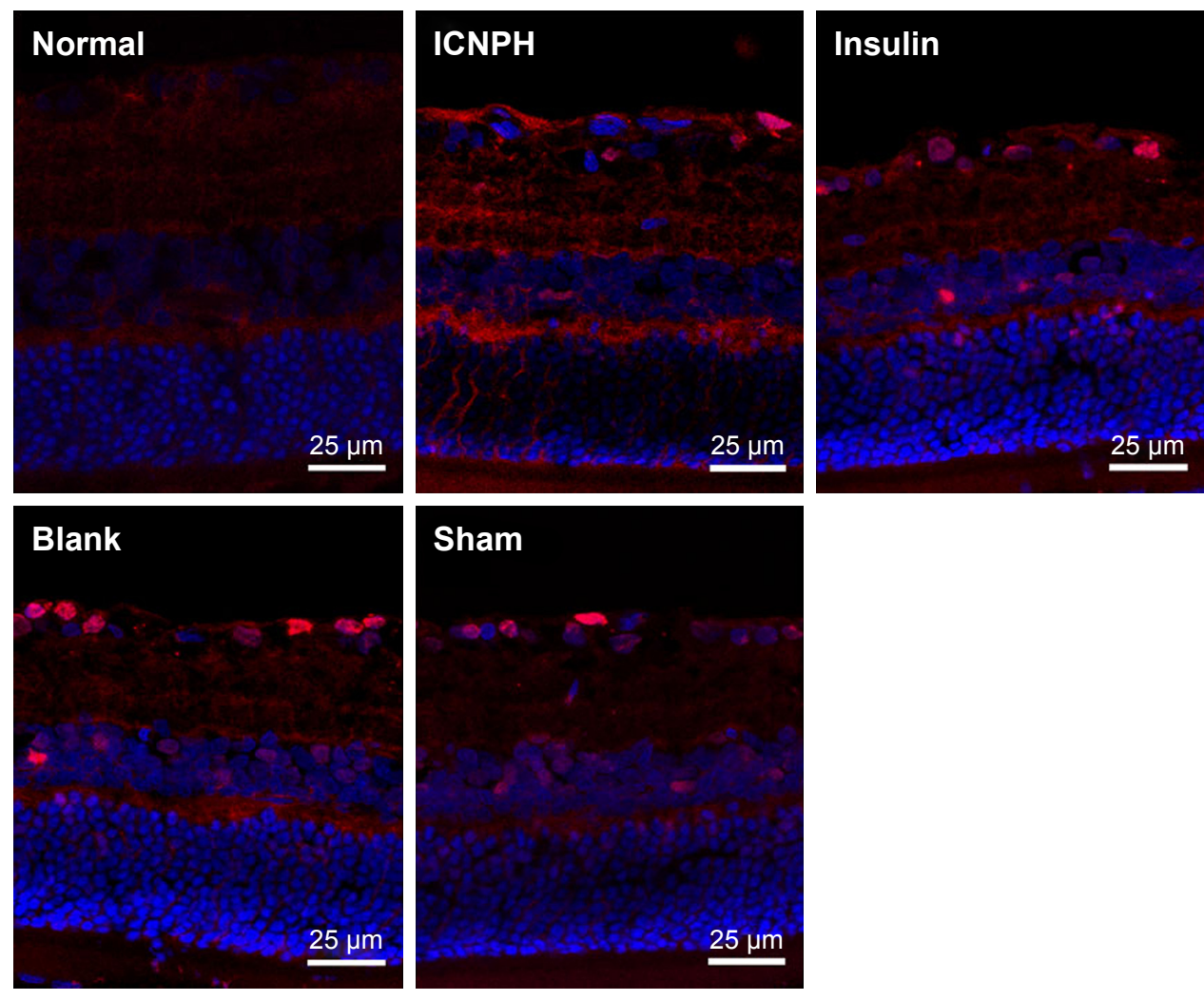

Figure 3 TUNEL staining for apoptotic cells in retinas at 2 weeks after subconjunctival injection.

Notes: Normal group: no injection with any drugs; ICNPH group: subconjunctival injection of $20 \mu \mathrm{L}$ ICNPH containing $80 \mu g$ insulin; Insulin group: subconjunctival injection of $80 \mu \mathrm{g}$ insulin in $20 \mu \mathrm{L}$ deionized water; Blank group: subconjunctival injection of $20 \mu \mathrm{L}$ blank nanosized hydrogel; Sham group: an empty syringe without any drugs was punctured into the conjunctiva of the diabetic rats.

Abbreviations: ICNPH, insulin-loaded chitosan nanoparticles/PLGA-PEG-PLGA composite hydrogel; PLGA-PEG-PLGA, poly(lactic-co-glycolic acid)-poly(ethylene glycol)poly(lactic-co-glycolic acid); TUNEL, terminal deoxynucleotidyltransferase-mediated dUTP nick end labeling.

among insulin, blank, and sham groups in terms of the extent of apoptosis.

\section{Expression of GFAP, VEGF, and Occludin in retina}

To further evaluate the neuroprotective effect of ICNPH on retina, we investigated the expression of GFAP and VEGF in retinal sections via immunofluorescence staining. Limited GFAP was localized on the fiber layer and in the vicinity of the inner limiting membrane in normal retinas (Figure 4A) while VEGF expression was significantly increased in the ganglion cell layer and also observed in the inner core layer, outer membrane, and pigment epithelium (Figure 4B). Compared with the normal control group, a slight increase of GFAP and VEGF expression was detected in the ICNPH group. In insulin, blank, and sham treatment groups, retinas exhibited remarkably higher levels of GFAP and VEGF, compared with the ICNPH group.

To further confirm the impact of ICNPH on the expressions of GFAP and VEGF, Western blot and real-time PCR assays, respectively, were conducted. Consistent with immunofluorescence findings, protein and mRNA levels of both GFAP and VEGF were significantly higher in insulin-, blank-, and sham-injected retinas compared with those in normal retina $(P<0.05, P<0.01)$. Notably, however, expression levels of these proteins in the ICNPH group were not significantly different from those in the normal group (Figure 5A and B).

Occludin is continuously expressed in the inner retinal vascular wall in normal retina, as shown in Figure $4 \mathrm{C}$. Expression of Occludin was significantly reduced in the retina of the sham group, as evident from immunofluorescence staining, Western blot, and real-time PCR data, similar to the insulin and blank groups ( $P<0.01$; Figures $4 \mathrm{C}$ and $5 \mathrm{C})$. In the ICNPH group, Occludin was downregulated, although the difference in expression was not significant, compared with the normal group.

\section{Discussion}

DR, one of the most serious complications of diabetes, is the third most common blinding eye disorder, affecting $\sim 60 \%-70 \%$ of diabetic patients within 15 years after onset. ${ }^{1}$ At present, 

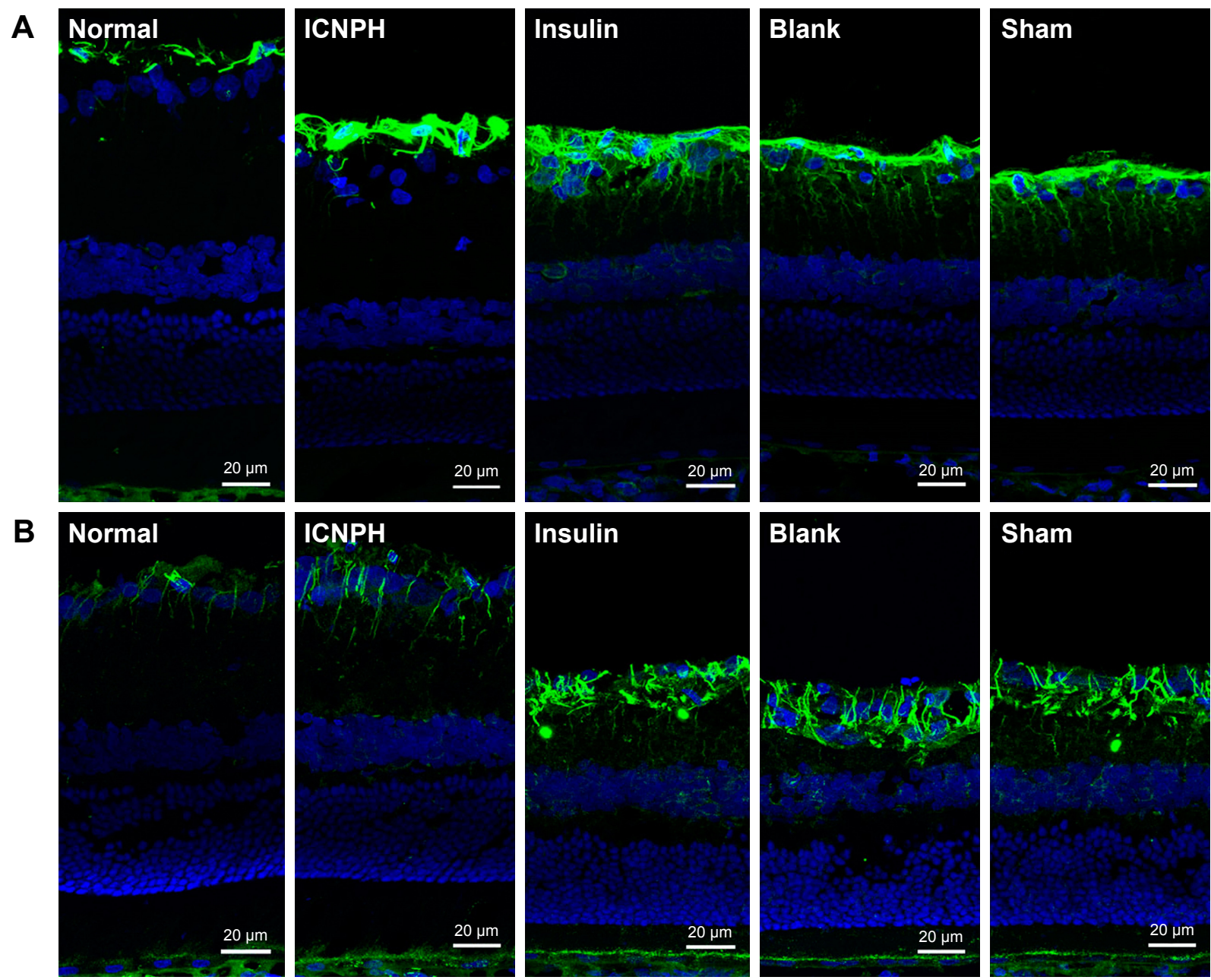

C Normal
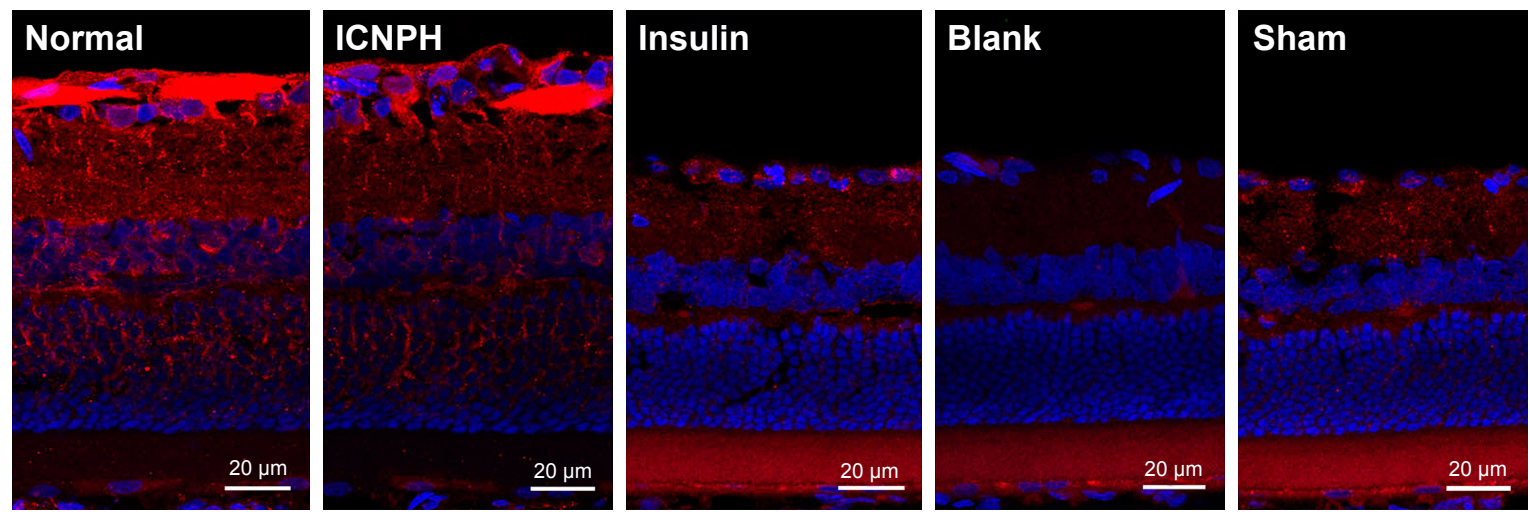

Figure 4 Immunofluorescence staining for GFAP, VEGF, and Occludin expression in retina at 2 weeks after subconjunctival injection.

Notes: (A) Immunofluorescence staining showing GFAP expression in retina in different groups. (B) Immunofluorescence staining showing VEGF expression in retina in different groups. (C) Immunofluorescence staining showing Occludin expression in retina in different groups. Normal group: no injection with any drugs; ICNPH group: subconjunctival injection of $20 \mu \mathrm{L}$ ICNPH containing $80 \mu \mathrm{g}$ insulin; Insulin group: subconjunctival injection of $80 \mu \mathrm{g}$ insulin in $20 \mu \mathrm{L}$ deionized water; Blank group: subconjunctival injection of $20 \mu \mathrm{L}$ blank nanosized hydrogel; Sham group: an empty syringe without any drugs was punctured into the conjunctiva of the diabetic rats.

Abbreviations: GFAP, glial fibrillary acidic protein; ICNPH, insulin-loaded chitosan nanoparticles/PLGA-PEG-PLGA composite hydrogel; PLGA-PEG-PLGA, poly(lactic-coglycolic acid)-poly(ethylene glycol)-poly(lactic-co-glycolic acid); VEGF, vascular endothelial growth factor.

local insulin therapy remains a key approach to prevent and inhibit DR progression, and the effectiveness has been widely recognized based on numerous basic experimental and clinical studies. ${ }^{13}$ To date, diverse insulin release technologies have been applied to treat retinal diseases, including nanoparticles, ${ }^{6}$ microspheres, ${ }^{7}$ and biodegradable hydrogels. ${ }^{8}$ Despite a number of therapeutic achievements with these methods, wide application in the clinic is not possible because of significant limitations, including short time of release, frequent injections, several toxic side effects, 
A
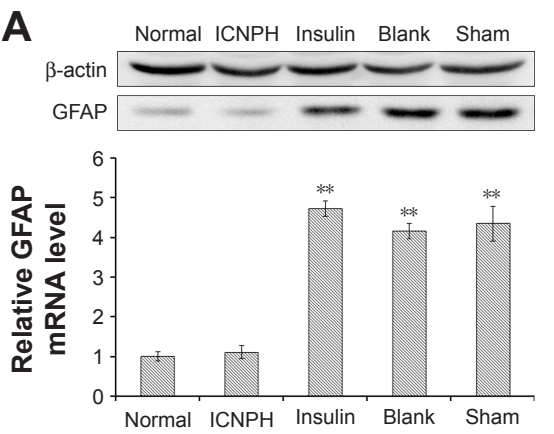

B
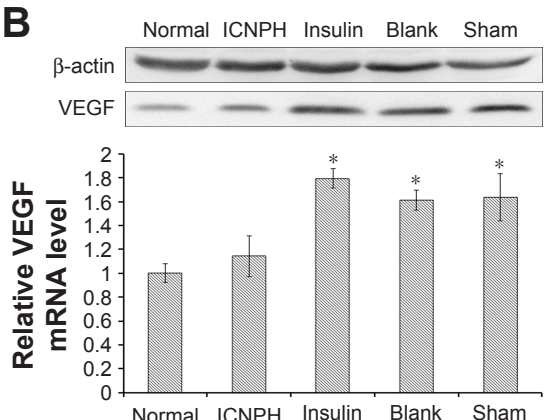

C
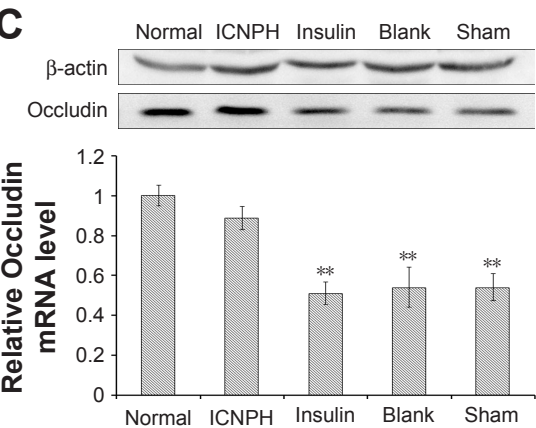

Figure 5 Western blot and real-time PCR assays for GFAP, VEGF, and Occludin expression levels in retina at 2 weeks after subconjunctival injection.

Notes: (A) The changes of GFAP expression at protein or mRNA levels in retinas in different groups. (B) The changes of VEGF expression at protein or mRNA levels in retinas in different groups. (C) The changes of Occludin expression at protein or mRNA levels in retinas in different groups. Normal group: no injection with any drugs; ICNPH group: subconjunctival injection of $20 \mu \mathrm{L} \mathrm{ICNPH} \mathrm{containing} 80 \mu \mathrm{g}$ insulin; Insulin group: subconjunctival injection of $80 \mu \mathrm{g}$ insulin in $20 \mu \mathrm{L}$ deionized water; Blank group: subconjunctival injection of $20 \mu \mathrm{L}$ blank nanosized hydrogel; Sham group: an empty syringe without any drugs was punctured into the conjunctiva of the diabetic rats. $* P<0.05 ; * * P<0.01$.

Abbreviations: GFAP, glial fibrillary acidic protein; ICNPH, insulin-loaded chitosan nanoparticles/PLGA-PEG-PLGA composite hydrogel; PLGA-PEG-PLGA, poly(lactic-coglycolic acid)-poly(ethylene glycol)-poly(lactic-co-glycolic acid); real-time PCR, real-time quantitative polymerase chain reaction; VEGF, vascular endothelial growth factor.

and damage to eyes. ${ }^{16}$ In the current study, a novel insulin delivery system, ICNPH, was developed, and its neuroprotective effect on DR through a single subconjunctival injection was investigated in a diabetic rat model.

DR at the early stages is generally accompanied by significant changes in retinal function, including increased retinal vascular permeability, loss of nerve cells, ${ }^{17}$ changes in ERG wave amplitude, ${ }^{18}$ and recession of visual sensitivity. ${ }^{19}$ Here, we performed binocular ERG of experimental rats at 4 weeks post subconjunctival injection to determine the influence of ICNPH on retinal function. ERG B-wave arises from the current flow along Müller cells in response to increased extracellular potassium ion concentrations associated with retinal circulation. ${ }^{20}$ In our experiments, ICNPH injection significantly reduced the decrease of scotopic B-wave amplitude in the diabetic rat compared with the insulin, blank, and sham treatments, suggesting the protective effect on retinal function. In the insulin group, the B-wave of the injected eye was almost the same with the fellow eye. One reason responsible for this might be that the amount of insulin injected once in the study was too high to exert its therapeutic effect. Another potential might be that the therapeutic level of insulin could not be sustained long after a single injection.

HE staining and TEM were employed to detect the retinal microstructure and ultrastructural changes in DR rats. Normal retinas exhibited thick tissues, intact basal membrane structure, and closely arranged RGCs. In insulin, blank, and sham groups, significant abnormalities in the retinal ultrastructure were observed, such as thinning of the retinal layers, decreased cell layer, rupture of retinal vessels and RGCs, and mitochondrial swelling. These changes were remarkably reduced in the ICNPH-injected group, indicating that ICNPH could effectively relieve the diabetes-induced damages to retinal tissues and cells. Cell apoptosis, one of the earliest and important features of DR, generally occurs in RGCs, endothelial cells, and astrocytes, ${ }^{21,22}$ and contributes to microaneurysms, acellular capillaries formation, blood retinal barrier breakdown, and retinal ischemia, subsequently promoting transition to proliferative DR. The results in the study showed the presence of TUNEL-positive cells in retinas of all groups of diabetic rats at 2 weeks post subconjunctival injection. Notably, significantly fewer apoptotic retinal cells were detected in the ICNPH group compared with the other injected groups. The results suggested the protective effect of ICNPH on DR. Meanwhile, the results might also reveal a desirable sustained release property of ICNPH in vitro, which facilitates release of insulin in a slow and durable manner, thus exerting a long-lasting protective effect on retina and reducing retinal cell death caused by long-term hyperglycemia.

As a metabolic disorder, diabetes can trigger the activation of transcription factors and augmentation of several vasoactive and growth factors, such as VEGF and GFAP, which further contribute to the initiation of chronic complications, resulting in structural and functional alterations in retina. ${ }^{23,24}$ In our study, only limited GFAP distributed on the fiber layer and vicinity of the inner limiting membrane was stained in normal retinas. A marked increase in the GFAP level was evident in the retinas of DR rats, compared with normal retinas. Whereas, the increased GFAP expression was significantly reduced in the ICNPH-administered group, compared with the insulin, blank, and sham groups. GFAP induced by activated glial cells in retinas is a nonspecific marker of retinal damage, ${ }^{25}$ considered a critical factor in RGC apoptosis. ${ }^{26}$ The results in the study clearly demonstrate the protective effects of ICNPH on glial activation in retina. A similar upregulation of VEGF expression was observed in 
retina of diabetic rats among insulin, blank, and sham groups. The expression level was remarkably lower in ICNPH group compared with the sham group. Previous studies have revealed that increased expression of VEGF in retina is associated with the progression of proliferative DR. ${ }^{27}$ A key role of VEGF in the pathogenesis of diabetic macular edema owns to its function in enhancing vascular permeability. ${ }^{28}$ The results indirectly suggested a protective effect of ICNPH on retinal vascular structure and permeability.

Occludin, an endothelial tight junction protein, is a critical protein responsible for connection of blood vessel walls. Shyong et $\mathrm{al}^{29}$ reported that development of diabetes results in significant downregulation of Occludin and disrupts its original distribution in retinal vascular endothelial cells. In our study, ICNPH treatment could significantly reduce the aberrant expression pattern of Occludin, demonstrating a protective effect of this delivery system against hyperglycemia-induced blood-retinal barrier destruction in retina.

\section{Conclusion}

Subconjunctival injection of ICNPH effectively protects retinal function, as revealed by ERG test, with less retinal microstructure and ultrastructure changes, reduced cell apoptosis, decreased GFAP and VEGF expressions, and increased Occludin expression, compared with rats receiving an injection of insulin, blank hydrogel, or sham treatment. The results demonstrate the neuroprotective effect of ICNPH on DR and suggest that the novel drug delivery system, ICNPH, is both feasible and effective through subconjunctival injection for the treatment of DR.

\section{Acknowledgments}

This work was supported by the National Natural Science Foundation of China (grant nos. 813008068, 81570842, 81770907, 81470613, 81100653, 81670835, and 81270989), Shanghai High Myopia Study Group, International Science and Technology Cooperation Foundation of Shanghai (grant no. 14430721100), Shanghai Talent Development Fund (grant no. 201604), Shanghai Youth Doctor Support Program (grant no. 2014118), and Outstanding Youth Medical Talents Program of Shanghai Health and Family Planning Commission (2017YQ011). The fund providers played no part in study design, data collection, analysis, decision to publish, or manuscript preparation.

\section{Disclosure}

The authors report no conflicts of interest in this work.

\section{References}

1. Kempen JH, O'Colmain BJ, Leske MC, et al. The prevalence of diabetic retinopathy among adults in the United States. Arch Ophthalmol. 2004; 122(4):552-563.

2. Diabetes Control and Complications Trial Research Group, Nathan DM, Genuth S, Lachin J, et al. The effect of intensive treatment of diabetes on the development and progression of long-term complications in insulindependent diabetes mellitus. $N$ Engl J Med. 1993;329(14):977-986.

3. White NH, Sun W, Cleary PA, et al. Prolonged effect of intensive therapy on the risk of retinopathy complications in patients with type 1 diabetes mellitus: 10 years after the Diabetes Control and Complications Trial. Arch Ophthalmol. 2008;126(12):1707-1715.

4. Cryer PE. Hypoglycemia is the limiting factor in the management of diabetes. Diabetes Metab Res Rev. 1999;15(1):42-46.

5. Khan MI, Barlow RB, Weinstock RS. Acute hypoglycemia decreases central retinal function in the human eye. Vision Res. 2011;51(14): 1623-1626.

6. Pan Y, Li YJ, Zhao HY, et al. Bioadhesive polysaccharide in protein delivery system: chitosan nanoparticles improve the intestinal absorption of insulin in vivo. Int J Pharm. 2002;249(1-2):139-147.

7. Varshosaz J, Sadrai H, Alinagari R. Nasal delivery of insulin using chitosan microspheres. J Microencapsul. 2004;21(7):761-774.

8. Misra GP, Singh RS, Aleman TS, Jacobson SG, Gardner TW, Lowe TL. Subconjunctivally implantable hydrogels with degradable and thermoresponsive properties for sustained release of insulin to the retina. Biomaterials. 2009;30(33):6541-6547.

9. Li X, Zheng X, Wei X, et al. A novel composite drug delivery system: honokiol nanoparticles in thermosensitive hydrogel based on chitosan. J Nanosci Nanotechnol. 2009;9(8):4586-4592.

10. Jeong B, Kim SW, Bae YH. Thermosensitive sol-gel reversible hydrogels. Adv Drug Deliv Rev. 2012;64(1):154-162.

11. Gong C, Shi S, Wu L, et al. Biodegradable in situ gel-forming controlled drug delivery system based on thermosensitive PCL-PEG-PCL hydrogel. Part 2: sol-gel-sol transition and drug delivery behavior. Acta Biomater. 2009;5(9):3358-3370.

12. Teles H, Vermonden T, Eggink G, Hennink WE, de Wolf FA. Hydrogels of collagen-inspired telechelic triblock copolymers for the sustained release of proteins. J Control Release. 2010;147(2):298-303.

13. Pitkänen L, Ranta VP, Moilanen H, Urtti A. Permeability of retinal pigment epithelium: effects of permeant molecular weight and lipophilicity. Invest Ophthalmol Vis Sci. 2005;46(2):641-646.

14. Lalloo A, Chao P, Hu P, Stein S, Sinko PJ. Pharmacokinetic and pharmacodynamic evaluation of a novel in situ forming poly(ethylene glycol)-based hydrogel for the controlled delivery of the camptothecins. J Control Release. 2006;112(3):333-342.

15. Paavola A, Kilpeläinen I, Yliruusi J, Rosenberg P. Controlled release injectable liposomal gel of ibuprofen for epidural analgesia. Int J Pharm. 2000;199(1):85-93.

16. Damgé $C$, Maincent $P$, Ubrich N. Oral delivery of insulin associated to polymeric nanoparticles in diabetic rats. J Control Release. 2007;117(2): 163-170.

17. Barber AJ, Gardner TW, Abcouwer SF. The significance of vascular and neural apoptosis to the pathology of diabetic retinopathy. Invest Ophthalmol Vis Sci. 2011;52(2):1156.

18. Han Y, Bearse MA, Schneck ME, Barez S, Jacobsen CH, Adams AJ. Multifocal electroretinogram delays predict sites of subsequent diabetic retinopathy. Invest Ophthalmol Vis Sci. 2004;45(3):948-954.

19. Ghirlanda G, di Leo MA, Caputo S, Cercone S, Greco AV. From functional to microvascular abnormalities in early diabetic retinopathy. Diabetes Metab Rev. 1997;13(1):15-35.

20. El-Hossary GG, El-Gohary AA, Ahmed NS, Mohamed AS, Mansour SM. Amelioration of chlorpyrifos induced retinal and renal toxicity by Vitamin D. Aust J Basic Appl Sci. 2009;3(3):2304-2314.

21. Singh AK, Gudehithlu KP, Patri S, et al. Impaired integration of endothelial progenitor cells in capillaries of diabetic wounds is reversible with vascular endothelial growth factor infusion. Transl Res. 2007; 149(5):282-291. 
22. Emanueli C, Graiani G, Salis MB, Gadau S, Desortes E, Madeddu P. Prophylactic gene therapy with human tissue kallikrein ameliorates limb ischemia recovery in type 1 diabetic mice. Diabetes. 2004;53(4): 1096-1103.

23. Khan ZA, Farhangkhoee H, Chakrabarti S. Towards newer molecular targets for chronic diabetic complications. Curr Vasc Pharmacol. 2006; 4(1):45-57.

24. Brownlee M. Biochemistry and molecular cell biology of diabetic complications. Nature. 2001;414(6865):813-820.

25. O'Callaghan JP. Assessment of neurotoxicity: use of glial fibrillary acidic protein as a biomarker. Biomed Environ Sci. 1991;4(1-2):197-206.

26. Woldemussie E, Wijono M, Ruiz G. Müller cell response to laserinduced increase in intraocular pressure in rats. Glia. 2004;47(2): 109-119.
27. Tretiach ML. Bovine models of human retinal disease: effect of perivascular cells on retinal endothelial cell permeability. 2005. Available from: https://ses.library.usyd.edu.au/handle/2123/1153. Accessed August 20, 2018.

28. Peters S. Angiopoietin-2 increases the permeability-enhancing effect of vascular endothelial growth factor (VEGF) in retinal vascular endothelial cells. 2004. Available from: https://www.egms.de/static/ de/meetings/dog2004/04dog612.shtml. Accessed August 20, 2018.

29. Shyong MP, Lee FL, Kuo PC, et al. Reduction of experimental diabetic vascular leakage by delivery of angiostatin with a recombinant adenoassociated virus vector. Mol Vis. 2007;13(15-16):133.
International Journal of Nanomedicine

\section{Publish your work in this journal}

The International Journal of Nanomedicine is an international, peerreviewed journal focusing on the application of nanotechnology in diagnostics, therapeutics, and drug delivery systems throughout the biomedical field. This journal is indexed on PubMed Central, MedLine, CAS, SciSearch $®$, Current Contents $\AA /$ Clinical Medicine,

\section{Dovepress}

Journal Citation Reports/Science Edition, EMBase, Scopus and the Elsevier Bibliographic databases. The manuscript management system is completely online and includes a very quick and fair peer-review system, which is all easy to use. Visit http://www.dovepress.com/ testimonials.php to read real quotes from published authors.

Submit your manuscript here: http://www.dovepress.com/international-journal-of-nanomedicine-journal 